\title{
Mechanical behavior of Mg subjected to strain path changes: experiments and modeling
}

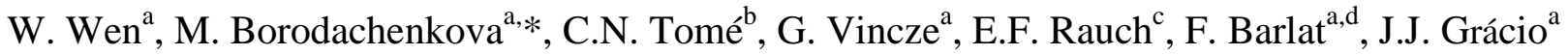 \\ ${ }^{a}$ Center for Mechanical Technology and Automation, Department of Mechanical Engineering, University \\ of Aveiro, 3810-193 Aveiro, Portugal \\ ${ }^{b}$ Materials Science and Technology Division - Los Alamos National Laboratory - Los Alamos, NM 87545 \\ USA \\ ${ }^{c}$ Science et Ingénierie des Matériaux et des Procédés - (CNRS UMR 5266) INPG-UJF, BP 46, 38402 \\ Saint Martin d'Hères cedex, France \\ ${ }^{d}$ Graduate Institute of Ferrous Technology (GIFT), Pohang University of Science and Technology \\ (POSTECH), 77 cheongam-ro, Nam-gu, Pohang, Gyeongbuk 790-784, Republic of Korea.
}

*Corresponding author: mborodachenkova@ua.pt

Tel. +351 234370 827, Fax. +351234370953

\begin{abstract}
Two-step tension tests with reloads along different directions are performed on rolled $\mathrm{Mg}$ alloy sheet at room temperature. The experimental yield stress at reloading is systematically lower than before unloading. Such a behavior is captured by a microstructure-based hardening model accounting for dislocation reversibility and back-stress. This formulation, embedded in the Visco-Plastic Self-Consistent (VPSC) model, links the dislocation density evolution throughout the deformation with hardening. The predicted results agree well with the experimental data in terms of flow stress response and texture evolution. The effects of texture anisotropy and backstress on the mechanical response during the strain path change are discussed.
\end{abstract}

Keywords: Crystallographic dislocation model, Microstructures, Strain path change, Polycrystalline material, Mechanical testing. 


\section{Introduction}

Magnesium alloys have low density and high specific strength and have been applied in lightweight structural parts of fuel efficient vehicles during the last two decades (Li et al., 2014; Mayama et al., 2011; Paliwal and Jung, 2014; Sánchez-Martín et al., 2014). However, the highly directional plastic properties of HCP crystals, and the fact that slip and twinning modes exhibit large differences in their activation stresses, makes the plastic response of HCP metals complex and very anisotropic (Agnew et al., 2001, 2005; Brown et al., 2005, 2012; Chapuis et al., 2014; Ma et al., 2012; Piao et al., 2012; Schoenfeld et al., 1995; Zhang et al., 2011). In particular, the restricted number of "soft" primary slip modes limit their formability at room temperature. HCP metals show better ductility and formability at higher temperature or moderate strain rates due to the low flow resistance and the activation of secondary slip systems (Jain and Agnew, 2007; Khan et al., 2011; Khosravani et al., 2013; Neil and Agnew, 2009; Oppedal et al., 2012; Proust et al., 2007, 2009; Wang et al., 2011, 2013).

Because most forming processes involve strain path changes, the present work investigates the mechanical behavior of $\mathrm{Mg}$ at room temperature associated with strain path changes. Usually, when a metallic material is plastically reloaded following a previous deformation path, it exhibits significant changes in the reloading yield stress and hardening evolution depending on the mode and direction of reloading (Beyerlein and Tomé, 2007; Knezevic et al., 2013; Rauch et al., 2007, 2011; Xue et al., 2007). This behavior has been characterized in many studies done on cubic materials (e.g. Lee et al. (2010) and Stout and Rollett (1990) for Copper; Rossiter et al. (2010) and Yoon et al. (2005) for Aluminum; Rauch et al. (2007, 2011) and Barlat et al. (2011) for low carbon steel; Schwartz et al. (2013) for stainless steel). Two features of materials being reloaded along a different strain path are: their yield stress at reload differs from the flow stress previous to unloading, and the subsequent flow stress evolution exhibits a relatively extended transition in the form of a flow plateau. The former is a generalized Bauschinger effect and takes place during the initial $1 \%$ or $2 \%$ reload strain. It is associated with the presence of internal back stresses which help activate mobile dislocations created during the pre-load. The latter effect can involve large strain intervals, and is related with the dissolution of the dislocation structure created during preload and its replacement by a new structure characteristic of the reload strain path. In previous work we concentrated in modeling the latter mechanism for BCC low-carbon steel (Rauch et al., 2007, 2011; Kitayama et al., 2013). Rauch et al. (2011) proposes a hardening model (RGVB model from now on) based on generation and annihilation laws for three different dislocation populations, namely, forward, reversible and latent dislocations. Strain path changes alter the character of those dislocations, and this leads to changes in the flow stress. Because the 
RGVB model is formulated for a continuum, it does not account explicitly for texture effects, and the information about path changes has to be introduced explicitly during the simulations. A crystallographic model based on RGVB ideas is proposed by Kitayama et al. (2013). The latter describes the generation and annihilation of forward and reversible dislocations in each crystallographic system of each grain. As a consequence, it accounts explicitly for the directionality and reversibility of slip, and for the hardening of individual systems in each grain. Such crystallographic model is implemented in the Visco-Plastic Self-Consistent (VPSC) polycrystals code, which accounts for grain interaction with the surroundings and also for texture effects and anisotropic plastic response. Here, we present a combined experimental and numerical study which extend the Kitayama et al. model to HCP Mg alloy AZ31, and which accounts for both, the short and the long range effects of pre-load onto reloading. We should note that the RGBV and the Kitayama et al. (2013) models do not account for the back-stress effects. In the present work, the back stress is accounted for by assuming that its effect is to lower the effective CRSS required to activate reversible dislocations in any given slip system.

In this work, a rolled Mg AZ31 sheet is preloaded in tension along the Rolling Direction (RD), and then reloaded in tension at different angles with respect to the RD. Stress strain response, texture evolution, and Lankford coefficient evolution are measured. The hardening model described above is used to interpret and predict the experimental data in terms of dislocation glide and activity in the basal and prism systems. Pyramidal slip and tensile twinning are allowed but do not make a relevant contribution to the mechanical response observed.

\section{Experimental Study}

Large dog-bone samples are cut from a rolled and annealed AZ31 Mg alloy sheet, with gauge length of $300 \mathrm{~mm}$ and width of $60 \mathrm{~mm}$, and then subjected to uniaxial tensile test for a pre-strain of $\varepsilon_{\mathrm{p}}=7 \%$. The pre-straining was carried out along the rolling direction (RD) in an Instron 4208 universal testing machine with a maximum load capacity of $150 \mathrm{kN}$. The cross-head speed (test speed) was $20 \mathrm{~mm} / \mathrm{min}$, which corresponds to an initial strain rate of $\dot{\varepsilon}=10^{-3} \mathrm{~s}^{-1}$. Reloading was performed by uniaxial tensile tests done on ASTM standard tensile specimens with $10 \mathrm{~mm}$ width cut from the pre-deformed material. The samples were cut at $0^{\circ}(\mathrm{RD}), 15^{\circ}, 30^{\circ}, 45^{\circ}, 60^{\circ}, 75^{\circ}$ and $90^{\circ}$ (TD) from the RD using as a guidance the striations left by rolling on the rolling plane. The main texture axes of the pre-deformed sample turned out to be tilted $9^{\circ}$ with respect to the RD striations, which explains the near coincidence between the $75^{\circ}$ and $90^{\circ}$ stress response, since they actually correspond to $84^{\circ}$ and $99^{\circ}$ with respect to the main texture axes. The Shimadzu AG$50 \mathrm{kN}$ tensile test machine was used for reloading tests and a videoextensometer ME 46 was used 
for strain measurement in two directions, namely longitudinal and transverse. All tests were performed at room temperature.

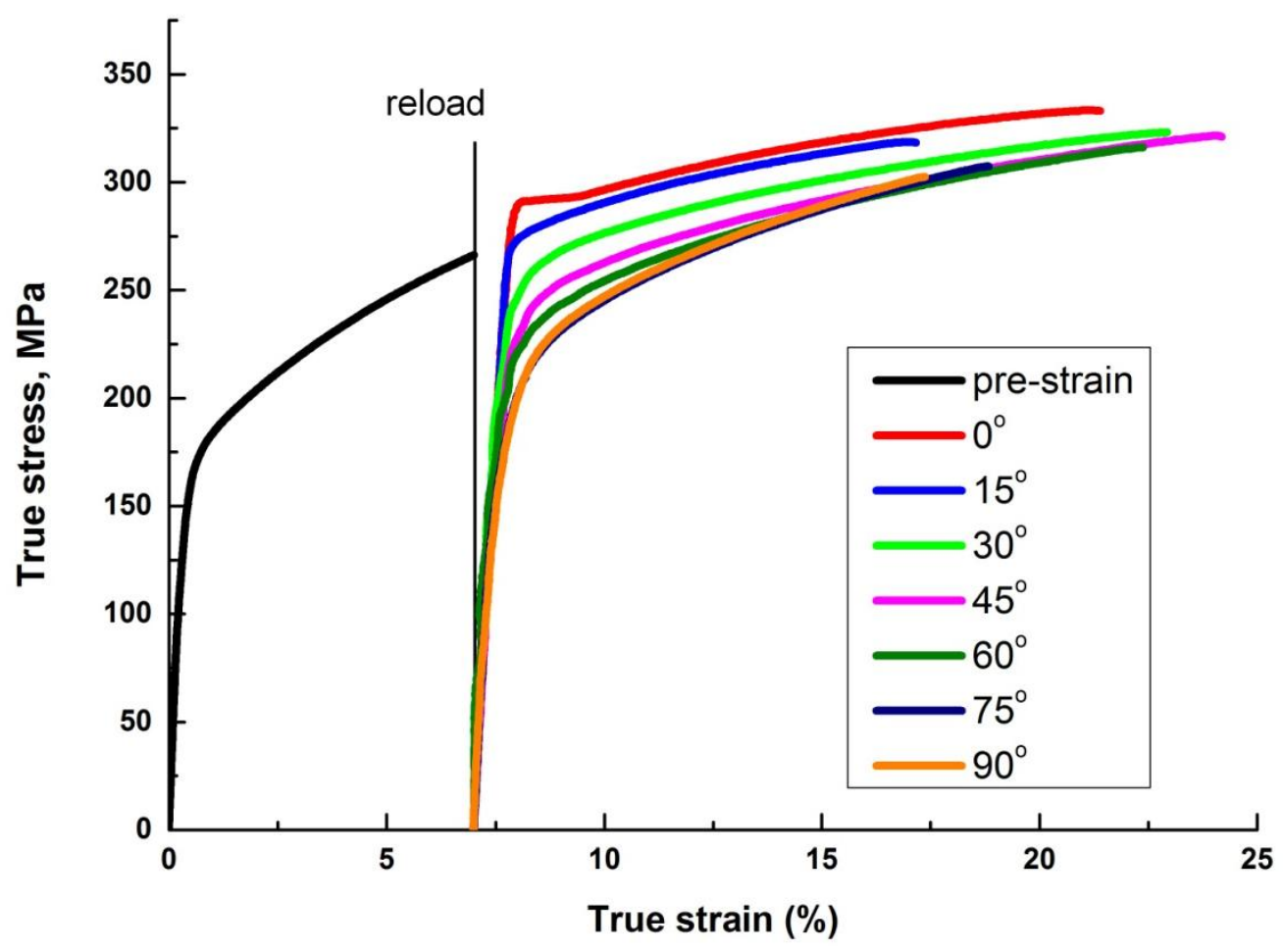

Fig. 1. Experimental true stress-true strain curves for tension-tension tests with different reloading angles from $\mathrm{RD}$ 
1

2

3

4

5

6

7

8

9

10

11

12

13

14

15

16

17

18

19

20

21

22

23

24

25

26

27

28

29

30

31

32

33

34

35

36

37

38

39

40

41

42

43

44

45

46

47

48

49

50

51

52

53

54

55

56

57

58

59

60

61

62

63

64

65
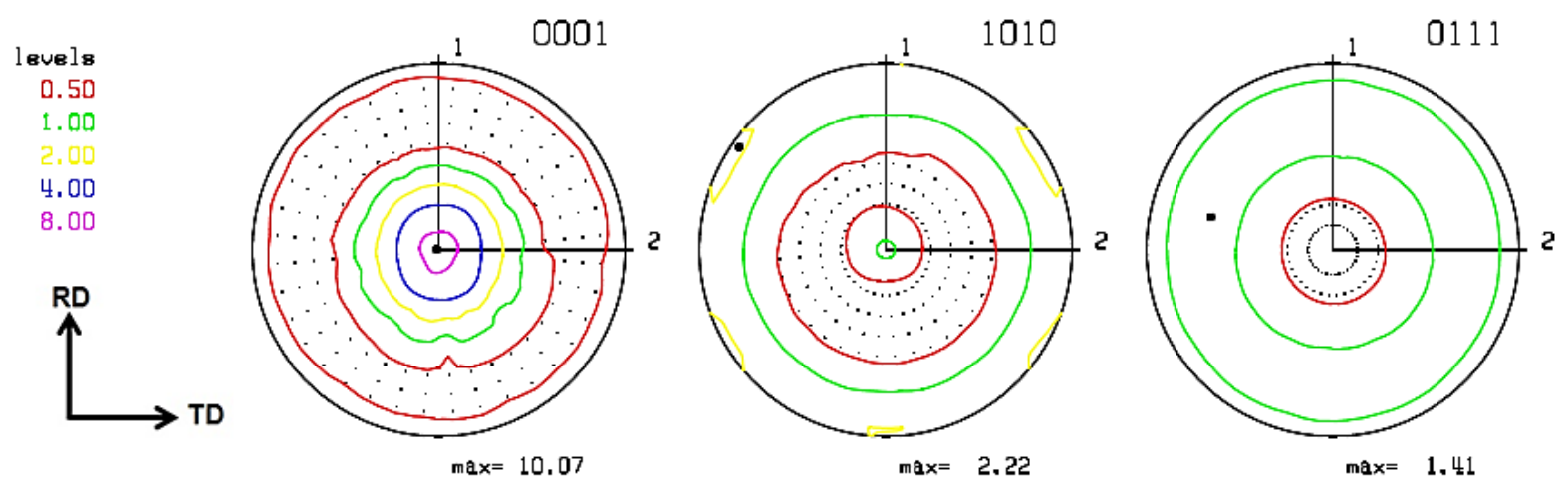

Fig. 2. Initial texture of rolled $\mathrm{Mg}$ sheet. 
For each reloading direction, three different samples were tested in order to validate the reproducibility of the results. The measured true strain-true stress curves are presented in Fig. 1. The yield stress at reloading decreases as the angle with respect to the preload direction increases.

The preferred crystallographic orientation (texture) was measured at the end of the different deformation tests by X-ray diffraction technique using a Philips X'pert diffractometer equipped with a texture goniometer $\mathrm{Cu} \mathrm{K \alpha}$ radiation was used. For texture analysis, the samples were polished at the mid-section along the thickness using 180, 400, 1000 grit $\mathrm{SiC}$ paper. Dedicated software MTEX Matlab quantitative texture analysis toolbox (Hielscher and Schaeben, 2008) was used to process the X-ray diffraction data into an Orientation Distribution File (ODF). The latter was processed to extract the discrete set of 2212 weighted grains that were used to represent the initial texture in our simulations. During these calculations no symmetry was imposed to the pole figures. The pole figures of the initial texture for the rolled AZ31 sheet are presented in Fig. 2 and represent a typical basal texture.

A relevant indicator of formability is the Lankford coefficient $(R)$ of the sheet, defined as the ratio $\varepsilon_{T} / \varepsilon_{N}$ between the transverse and the through thickness strain components associated with a tensile test. In this work we follow the evolution of $R$ with strain for all the tests performed. We do so by measuring the transverse strain $\varepsilon_{T}$. The through thickness strain is calculated using the measured tensile strain $\varepsilon_{L}$ and the volume conservation of plastic strain as $\varepsilon_{N}=-\left(\varepsilon_{L}+\varepsilon_{T}\right)$

\section{Modeling}

\subsection{Crystallographic RGBV model}

The crystallographic RGBV model allows tracking the dislocation density evolution for each slip system in each grain throughout the plastic deformation process. The dislocations can be accumulated during the pre-strain and then erased progressively after the strain path change. This model (Kitayama et al, 2013), embedded in the VPSC framework (Lebensohn and Tomé, 1993; Lebensohn et al., 2007), is able to identify the reversals of shear strain in each slip system of each grain which are usually associated with changes of the strain path, but may also be induced by grain reorientation during large plastic deformation. In this work, we assume that the CRSS is depends on the evolution of dislocation density and the back-stress. The contribution of backstress $\left(\Delta \tau_{B}^{s}\right)$ is introduced as a correction term of CRSS which is activated when a shear strain reversal on the system takes place. Thus, the CRSS on the slip system $s\left(\tau^{s}\right)$ can be expressed

$$
\tau^{s}=\tau_{d}^{s}+\Delta \tau_{B}^{s}
$$


The contribution of dislocation accumulation/annihilation on the CRSS $\left(\tau_{d}^{s}\right)$ is calculated

through the usual Taylor law. In order to describe correctly the latent hardening associated with dislocation-dislocation interactions within the HCP structure, the complex form of Taylor law (Cáceres and Blake, 2007; Cáceres and Lukáč, 2013; Capolungo, 2011; Kubin et al., 2008; Peeters et al., 2000, 2001) is used in this work instead of simpler one used by Kitayama et al. (2013) for BCC steel:

$$
\tau_{d}^{s}=\tau_{0}^{s}+\mu b \sqrt{\alpha^{s s} \rho^{s}+\sum_{s \neq s^{\prime}} \alpha^{s s^{\prime}} \rho^{s^{\prime}}}
$$

where $\tau_{0}^{s}$ is the initial CRSS, $\mu, b$ and $\rho^{s}$ denote the shear modulus, the magnitude of the Burgers vector and the total dislocation density for slip system $s$, respectively. $\alpha^{s s^{\prime}}$ is the latent hardening matrix.

For details of the hardening model employed here the reader is referred to Kitayama et al. (2013). The dislocation density on each slip system is comprised of forward and reversible dislocations. The reversible dislocations will be recombined if the shear on the slip system is reversed, which is in fact a mechanism of dislocation annihilation. In order to account for the effect of reversible dislocations in the simulation, each slip system is split into two systems $s^{+}$and $s^{-}$, which correspond to the activation of the slip system $s$ on the positive and negative directions of the Burgers vector (arbitrarily defined). Therefore, the dislocation density on the slip system $s$ is presented as:

$\rho^{s}=\rho_{\text {for }}^{s}+\rho_{\text {rev }}^{s+}+\rho_{\text {rev }}^{s-}$

here $\rho^{s}$ denotes the total dislocation density on slip system s. $\rho_{\text {for }}^{s}$ is the forward dislocation density. $\rho_{\text {rev }}^{s+}$ and $\rho_{\text {rev }}^{s-}$ represent the reversible dislocation density on $s^{+}$and $s^{-}$, respectively

The total dislocation density in one grain is given by

$\rho=\sum_{s} \rho^{s}=\sum_{s}\left(\rho_{\text {for }}^{s}+\rho_{\text {rev }}^{s+}+\rho_{\text {rev }}^{s-}\right)$

Upon generation, a fraction $p$ of the dislocations generated is assumed to be reversible.

$$
\begin{aligned}
& d \rho_{\text {for }}^{s}=(1-p) d \rho^{s} \\
& d \rho_{\text {rev }}^{s}=p d \rho^{s}
\end{aligned}
$$


The reversibility fraction $p$ varies with accumulated dislocation density. All dislocations

here $\rho_{d e b}=\int_{0}^{\varepsilon} \rho d \Gamma$ is a rough measure of the density of dislocations that become locked upon reaction and will not annihilate or be reversed. Its increase progressively decreases reversibility. $d \Gamma=\sum_{s}\left|d \gamma^{s}\right|$ is the total shear increment in one grain; $\rho_{\text {infl }}=\frac{\rho_{\text {max }}+\rho_{\min }}{2}, \rho_{\max }$ and $\rho_{\text {min }}$ are characteristic densities which can be regarded as material properties (Kitayama et al., 2013).

We define a dislocation mean free path $\Lambda$ :

$$
\frac{1}{\Lambda}=\frac{\sqrt{\rho}}{K}+\frac{1}{D}
$$

with $K$ the number of forest dislocations that a moving segment is able to cross before being trapped by the obstacles. $D$ is the average grain size.

For the crystallographic RGBV model, if the slip system is activated on the positive direction $\left(d \gamma^{s+}>0\right.$ and $\left.d \gamma^{s-}=0\right)$, the increment of $\rho_{\text {for }}^{s}$ and $\rho_{r e v}^{s+}$ are calculated by the Kocks-Mecking law (Kocks and Mecking, 2003) with both a storage term and a recovery term:

$$
\begin{aligned}
& d \rho_{f o r}^{s}=(1-p) \frac{d \gamma^{s+}}{b \Lambda}-f \rho_{f o r}^{s} d \Gamma \\
& d \rho_{r e v}^{s+}=p \frac{d \gamma^{s+}}{b \Lambda}-f \rho_{r e v}^{s+} d \Gamma
\end{aligned}
$$

Here, $d \Gamma$ is used in the recovery term instead of $d \gamma^{s+}$ because any active slip system can in principle affect the recombination in $s$. At the same time, the recombination of $\rho_{r e v}^{s-}$, which may have accumulated before the shear is reversed, is given by:

$$
d \rho_{\text {rev }}^{s-}=-\frac{1}{b \Lambda}\left(\frac{\rho_{r e v}^{s-}}{\rho_{0}^{s}}\right)^{m} d \gamma^{s+}
$$


The parameter $m$ was not originally proposed in the formulation of Kitayama et al (2013). It is introduced in this work to provide a faster rate of dislocation recombination at the beginning of the reversal process, when loosely pinned dislocations are reversed, and a slower rate at the end, when strongly pinned dislocations are activated. The activation of slip systems on positive and negative directions are equivalent. Thus, the expressions for dislocation density evolution when $s^{-}$is activated $\left(d \gamma^{s-}>0\right.$ and $\left.d \gamma^{s+}=0\right)$ are:

$$
\begin{aligned}
& d \rho_{\text {for }}^{s}=(1-p) \frac{d \gamma^{s-}}{b \Lambda}-f \rho_{f o r}^{s} d \Gamma \\
& d \rho_{r e v}^{s+}=-\frac{1}{b \Lambda}\left(\frac{\rho_{r e v}^{s+}}{\rho_{0}^{s}}\right)^{m} d \gamma^{s-} \\
& d \rho_{r e v}^{s-}=p \frac{d \gamma^{s-}}{b \Lambda}-f \rho_{r e v}^{s-} d \Gamma
\end{aligned}
$$

\subsection{Back-stress effects}

Besides the dislocation accumulation/annihilation, the hardening evolution during strain path change is also affected by back-stress acting on dislocations, an effect not accounted for by Kitayama et al. (2013). During the forward loading, a dislocation substructure is generated in the material, which includes the fraction of loosely tangled and reversible dislocation segments that are taken into account in the previous section. The substructure is also known to be associated to polarized back stresses (Essmann and Mughrabi, 1979; Mughrabi et al., 1986). In particular a heterogeneous distribution of dislocations leads to long range intragranular stresses that rapidly exceed the intergranular residual stresses related to strain incompatibilities (Feaugas, 1999). Consequently, it is reasonable to associate the residual stresses to the current state of the substructure.

At unloading only a small fraction of these stresses are released. This is because a large fraction of the stresses is related to more or less well-formed dislocation pile-ups that are trapped in the cell walls. The progressive untangling of previously stored dislocations upon strain path change favour the decrease of the residual stresses inherited from the prestrain. The trend is particularly sensitive when the strain is reversed leading to the well-known Bauschinger effect (Bauschinger, 1881).

In the present work, we account for the effect of back-stress on each slip system by assuming a direct relationship with the structural component that is also decreasing upon reloading, i.e.: the reversible dislocations accumulated during the pre-strain. For sake of simplicity, a linear 
dependence is considered. As a consequence, the resolved shear stress acting on the dislocations is corrected as follows:

$$
\begin{aligned}
& \Delta \tau_{B}^{s}=-\tau_{d}^{s} f_{B}^{s}\left(\frac{\rho_{\text {rev }}^{s-}}{\rho_{\text {total }}}\right) \quad \text { if } d \gamma^{+}>0 \\
& \Delta \tau_{B}^{s}=-\tau_{d}^{s} f_{B}^{s}\left(\frac{\rho_{\text {rev }}^{s+}}{\rho_{\text {total }}}\right) \quad \text { if } d \gamma^{-}>0
\end{aligned}
$$

therefore, the effective CRSS is expressed as:

$$
\tau^{s}=\tau_{d}^{s}+\Delta \tau_{B}^{s}=\tau_{d}^{s}\left[1-f_{B}^{s}\left(\frac{\rho_{r e v}^{s-(+)}}{\rho_{\text {total }}}\right)\right] \text { if } d \gamma^{+(-)}>0
$$

During the forward loading, reversible dislocations are generated. When the shear on slip system $s$ is reversed, the effective CRSS for the opposite system decreases by an amount given by Eq. 11. The resulting CRSS is used in Eq. 13 below. By construction, the residual stresses are directly related to the reversible dislocations density. Since according to Eqs. 9 and 10 the reversible dislocations recombine during reloading for strain path change, their effect on the effective CRSS decreases gradually and the back stresses vanish. $f_{B}^{s}$ in Eq. 11 is the scaling factor that weights the link between the reversible dislocation and back-stresses.

It should be noted that the crystallographic RGBV model only describes the hardening evolution of slip systems. The CRSS of each twinning system is considered as constant throughout the deformation process. The influence of this rough approximation is negligible since the activation of tensile twinning is limited for the loading conditions considered in this work (see section 5).

\subsection{Brief description of VPSC model}

The crystallographic RGBV model is embedded in the VPSC framework which allows to track the orientation of each grain in the polycrystals during the deformation. In this section, the VPSC model is briefly introduced. The detailed description of this model can be found in Lebensohn et al. (2007) and Wang et al. (2010). The self-consistent type crystal plasticity model treats each grain or grain group as an inhomogeneous inclusion embedded in the "homogeneous effective medium" (HEM) which has the average properties of the aggregate. The macroscopic response of the polycrystals results from the contribution of each grain. At the single crystal level, the strain rate is calculated according to the slip/twinning activations: 
$d^{g}=\sum_{s} m^{s} \dot{\gamma}^{s}=\dot{\gamma}_{0} \sum_{s} m^{s}\left(\frac{m^{s} \sigma^{g}}{\tau^{s}}\right)^{n}$

here, $m^{s}$ is the symmetric part of Schmid tensor and $\dot{\gamma}^{s}$ is the shear rate. $\dot{\gamma}_{0}$ denotes a normalized shear rate. $n$ is the inverse strain-rate sensitivity. $\tau^{s}$ is the CRSS which is determined by the RGBV model in this work. The relationships between strain-rate and stress for single crystal and for the aggregate are written in a linearized form as:

$d^{g}=M^{g} \sigma^{g}+d_{0}^{g}$
$D=\bar{M} \Sigma+D_{0}$

in Eq. $14, M^{g}$ and $\bar{M}$ are the local and macroscopic compliance tensors. $d_{0}^{g}$ and $D_{0}$ are the back-extrapolated terms for the grain and for the aggregate, respectively. The inclusion formalism provides the interaction between a grain and the aggregate in terms of local and average stress and strain rate, as:

$\left(d^{g}-D\right)=-\tilde{M}\left(\sigma^{g}-\Sigma\right)$

where

$\tilde{M}=n^{e f f}(I-E)^{-1}: E: \bar{M}^{\text {secant }}$

$d^{g}$ and $\sigma^{g}$ denote the deviatoric strain-rate and stress at the single crystal level. $D$ and $\Sigma$ are the macroscopic strain-rate and stress, respectively. $E$ represents the Eshelby tensor for a given grain. $\bar{M}^{\text {secant }}$ is the macroscopic visco-plastic compliance tensor for the Secant case. The parameter $n^{\text {eff }}$ describes the visco-plastic compliance of the inclusion-matrix interactions $n^{e f f}=0$ for a Taylor case, $n^{e f f}=1$ for a Secant case and $n^{e f f}=n$ for a Tangent case (Lebensohn and Tomé, 1993; Lebensohn et al., 2007). In this work we adopt the Tangent interaction with $n=20$.

In the present work, the Predominant Twin Reorientation (PTR) scheme (Tomé et al., 1991) is utilized in the VPSC framework to describe the twinning orientation. At each deformation step, the PTR scheme defines the associated volume fraction for each twinning system of one grain as $V^{t, g}=\gamma^{t, g} / S^{t}$ where $S^{t}$ is the characteristic twin shear $\left(S^{t}=0.13\right.$ for $\left.\mathrm{Mg}\right)$. The twinning system in this grain with the highest associated volume fraction is identified as the predominant twin system (PTS). At each incremental step, a grain is picked randomly, and the grain will be fully reoriented if the accumulated twin fraction exceeds a threshold value which is defined as: 


$$
V^{t h}=A^{t h 1}+A^{t h 2} \frac{V^{e f f}}{V^{a c c}}
$$

$V^{\text {eff }}$ represents the volume fraction of the grains that are already reoriented, and the values of $A^{t h 1}$ and $A^{t h 2}$ can be determined according to the polycrystals response. $V^{a c c}$ is the sum of $V^{t, g}$ over all the twinning systems and all grains. Once the condition is fulfilled, the grain will be completely reoriented and only the PTS is considered in the reorientation. This process will be repeated until either all grains are randomly picked or the $V^{\text {eff }}$ exceeds the sum of $V^{t, g}$ in this grain. As mentioned before, while for completeness we allow for possible twin activity, in the tests being simulated there is not a significant contribution of twinning to deformation.

\section{Simulation condition}

The simulation study is preformed using the modeling framework described in section 3 . The initial texture with 2122 orientations (Fig. 2) is utilized as input to the VPSC model. The tangent interaction is chosen and the inverse strain-rate sensitivity $n$ is taken as 20 . The $\{0001\}\langle 11 \overline{2} 0\rangle$ basal slip, $\{10 \overline{1} 0\}\langle 11 \overline{2} 0\rangle$ prismatic slip, $\{10 \overline{1} 0\}\langle 11 \overline{2} 3\rangle$ pyramidal slip and $\{10 \overline{1} 2\}\langle 10 \overline{1} 1\rangle$ tensile twinning are assumed to be activated in the simulations. Sequential simulations are carried out to predict the strain path change, which means that the simulated deformation states at the end of pre-strain process are considered as the initial states of reloading stage. The initial forward and reversible dislocation density for each slip system is assumed to be 0 since the tests are carried out on annealed Mg sheet. The effect of a small initial dislocation density in the annealed material is accounted for by the starting value of $\tau_{0}^{s}$ assigned to each system. Mimicking the experimental process described in section 2 a tensile test along a direction with $9^{\circ}$ deviation from $\mathrm{RD}$ is simulated up to $7 \%$ strain, followed by tension along the different reloading directions. The tensile tests before and after reloading are simulated under mixed stress and strain-rate boundary conditions, which enforce zero lateral stresses and shear in the RD-TD plane. The macroscopic velocity gradient $\bar{L}$ and Cauchy stress tensor $\Sigma$ enforced in the simulation adopt the form:

$$
\bar{L}=\frac{\partial \bar{U}}{\partial \bar{X}}=\left[\begin{array}{lll}
1 & ? & 0 \\
0 & ? & ? \\
0 & 0 & ?
\end{array}\right] \text { and } \Sigma=\left[\begin{array}{lll}
? & 0 & ? \\
& 0 & 0 \\
& & 0
\end{array}\right]
$$

Allowing for free displacement of the sample transverse surfaces not only captures the real conditions of the tests, but is essential for predicting the Lankford coefficient. The latent 
hardening matrix $\alpha^{s s^{\prime}}$ in Eq. 2 describes the dislocation-dislocation interactions between slip

\section{Results and discussion}

Under the simulation condition described in section 4, the predicted results in terms of stressstrain response, slip/twinning activity, dislocation density evolution, texture and Lankford coefficients, are presented in this section and compared with experiments.

The predicted stress-strain response of $\mathrm{Mg}$ alloy is shown in Fig. 3. Notice that these results are obtained using a visco-plastic modeling framework, which does not capture the elastic behavior and the elasto-plastic transition. In order to compare experimental and predicted stress-strain curves, the elastic part of the experimental curves is removed from the results. The measured curves show that the yield stress at reloading decreases as the reloading angle increases. Such a behavior is captured well in the prediction. While the alignment of the tensile axis relative to texture will affect the flow curve (average Taylor factor), the drop in flow stress during the initial reloading strain is mainly an effect of the back-stress as discussed later in connection with Fig. 8.

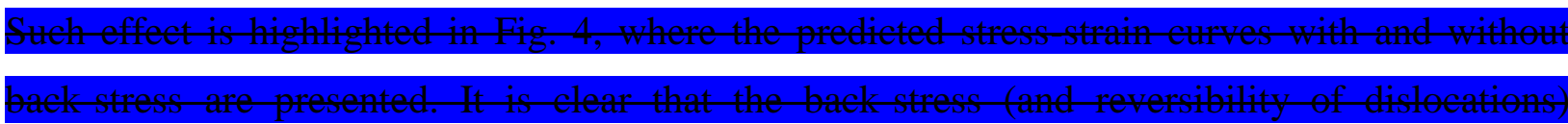

mereases for higher reloading angle, which is reasonable according to its definition.-The measured and predicted stress-strain responses for $75^{\circ}$ and $90^{\circ}$ reloading angles with respect to the RD almost overlap (Fig. 3) because these two reloading direction correspond to $84^{\circ}$ and $99^{\circ}$ from the actual pre-loading tensile axis (see section 2 and 4), which makes the related changes of strain path practically equivalent.

The predicted textures after pre-strain and after 13\% reloading are shown in Fig. 4 and compared with experiments. The initial texture exhibits a basal component which is typical for rolled $\mathrm{Mg}$ 
sheet, plus a weak fiber perpendicular to the ND (Fig. 2). After 7\% pre-strain (Fig. 4a), the basal

Table 1. Values of the latent hardening matrix $\alpha^{s s^{\prime}}$ for various slip/slip interactions

\begin{tabular}{|l|c|}
\hline \multicolumn{1}{|c|}{ Interaction } & Value \\
\hline Basal self-interaction & 0.8 \\
\hline Prismatic self-interaction & 0.45 \\
\hline coplanar Basal / Basal & 0.6 \\
\hline collinear Basal / Prismatic & 0.71 \\
\hline collinear Prismatic / Basal & 0.63 \\
\hline non-collinear Prismatic / Basal & 0.065 \\
\hline Prismatic / Prismatic & 0.04 \\
\hline non-collinear Basal / Prismatic & 0.06 \\
\hline
\end{tabular}


Table 2. Parameters of the RGBV model and PTR scheme for Mg alloy.

\begin{tabular}{|l|c|}
\hline \multicolumn{1}{|c|}{ Parameters } & Values \\
\hline$\mu$ (Elastic shear modulus) & $17 \mathrm{GPa}$ \\
\hline$b$ (Burgers vector) & $3.21 \cdot 10^{-10} \mathrm{~m}$ (prismatic), $3.21 \cdot 10^{-10} \mathrm{~m}$ (basal), \\
$6.077 \cdot 10^{-10} \mathrm{~m}($ pyramidal)
\end{tabular}




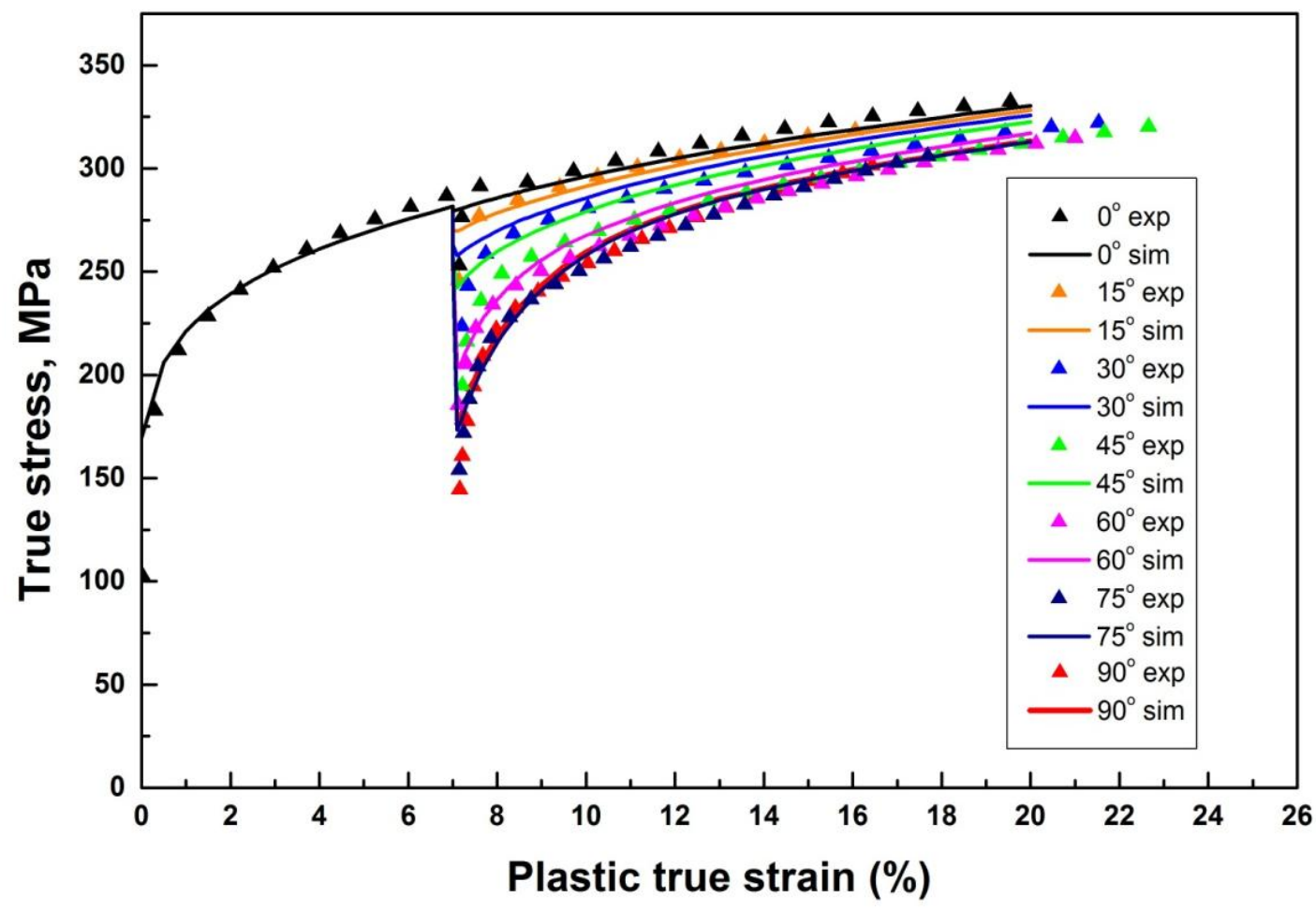

Fig. 3. Comparison of predicted and measured true strain-true stress curves for different 
a)

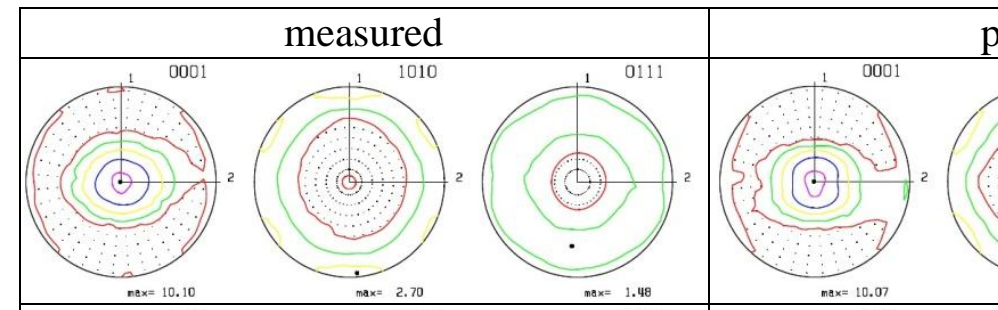

b)

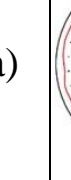
predicted

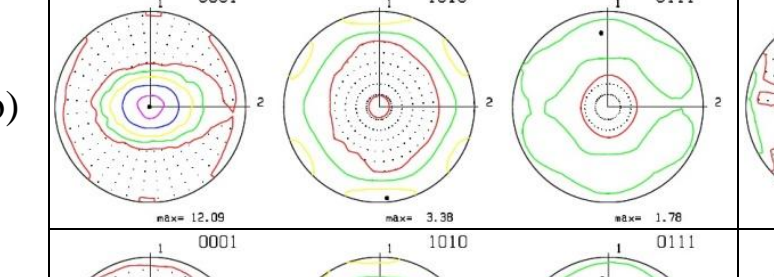

c)

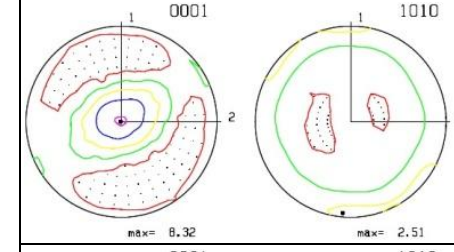

d)

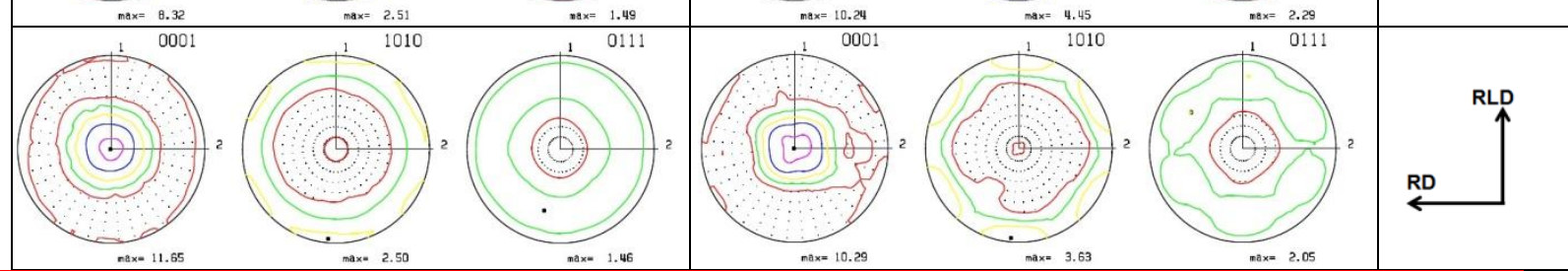

Fig. 4. Predicted and experimental texture after $7 \%$ pre-strain (a) and after $13 \%$ reloading for $0^{\circ}$

(b), $45^{\circ}$ (c) and $90^{\circ}$ (d) from RD. PLD=Pre-loading direction. RLD=reloading direction. Intensity 
The relative slip/twinning activities for $0^{\circ}, 30^{\circ}, 60^{\circ}$ and $90^{\circ}$ are shown in Fig. 5. The simulations et al., 2010). The tensile twinning systems show also little activity because, according to the measured texture, the loading condition in this work is mainly tension perpendicular to c-axis, which is not in favor of the considered twinning systems (Aghababaei and Joshi, 2014; Wang and Huang, 2003). The activity changes observed at the beginning of reloading should be considered as a coupled effect of texture anisotropy and reversal-related mechanisms. First, the back-stress can affect the slip activities via the effective CRSS associated with the dislocation substructure generated during the pre-loading. The activation of back-stress leads to a drop for the relative activity of the basal slip. Second, the texture evolution (Figs. 2 and 4) shows that some of the grains tend to rotate towards TD and the (0001) pole figure seems to be "elongated". When the tensile reloading direction is close to $\mathrm{TD}$, these grains are in favourable position to activate basal slip. The evolution of reversible and forward dislocation densities for different reloading directions is shown in Fig. 6. In the simulation, the forward and reversible dislocation generation is controlled by the reversibility fraction $p$ (Eqs. 5 and 6) which remains close to 1 at small strain and then gradually decreases to 0 as the strain increases. In the work of Kitayama et al., (2013), the forward dislocations appear after $40 \%$ shear strain. At the strain levels of our simulations nearly all the dislocations are reversible and, in addition, their density changes only slightly within the initial 2\% reloading strain (Fig 6c, 6d). Considering that the drop in yield stress is substantial at reloading, the effect has to be attributed to the back-stress associated with these dislocations.

The Lankford coefficient, defined in Section 2 as the ratio $R=\varepsilon_{T} / \varepsilon_{N}$ between the transverse and the through thickness strain components associated with a tensile test, is a measure of the forming capability of the rolled plate. It has been shown to be very sensitive to details of the constitutive model used to describe plasticity (Wang et al, 2010; Bertin et al, 2014). The Lankford coefficient was measured in this work for all the reloads and as a function of strain. It is reported in Fig. 7, and compared with the evolution predicted by our polycrystal simulations. The $90^{\circ}$ case is nearly identical to the $75^{\circ}$ case and is not depicted here. The experimentally measured strains are not reliable within the initial 3\% tensile pre-load or 3\% tensile reloads but, beyond those intervals, it is clear that $R$ increases monotonically with strain, indicating that the plate is hardening faster (and deforming less) along the ND. The rate of increase in R with strain is underestimated by the quantitative predictions for the $0^{\circ}$ to $45^{\circ}$ cases, while the uniform behaviour measured for the $60^{\circ}$ to $90^{\circ}$ cases is captured by the model. 

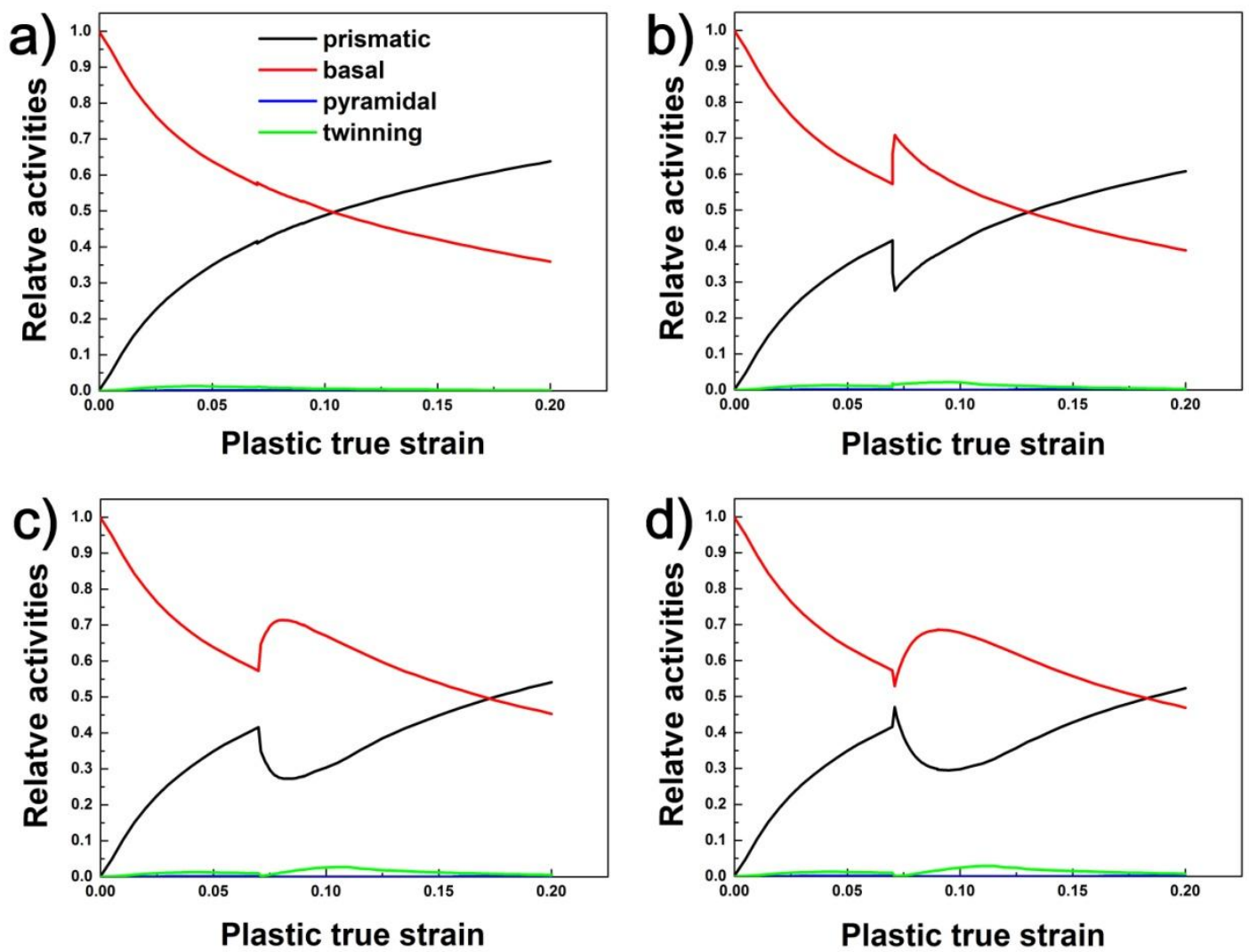

Fig. 5. Relative slip/twinning activities for the reloading along $0^{\circ}$ (a), $30^{\circ}$ (b), $60^{\circ}$ (c) and $90^{\circ}$ (d) 

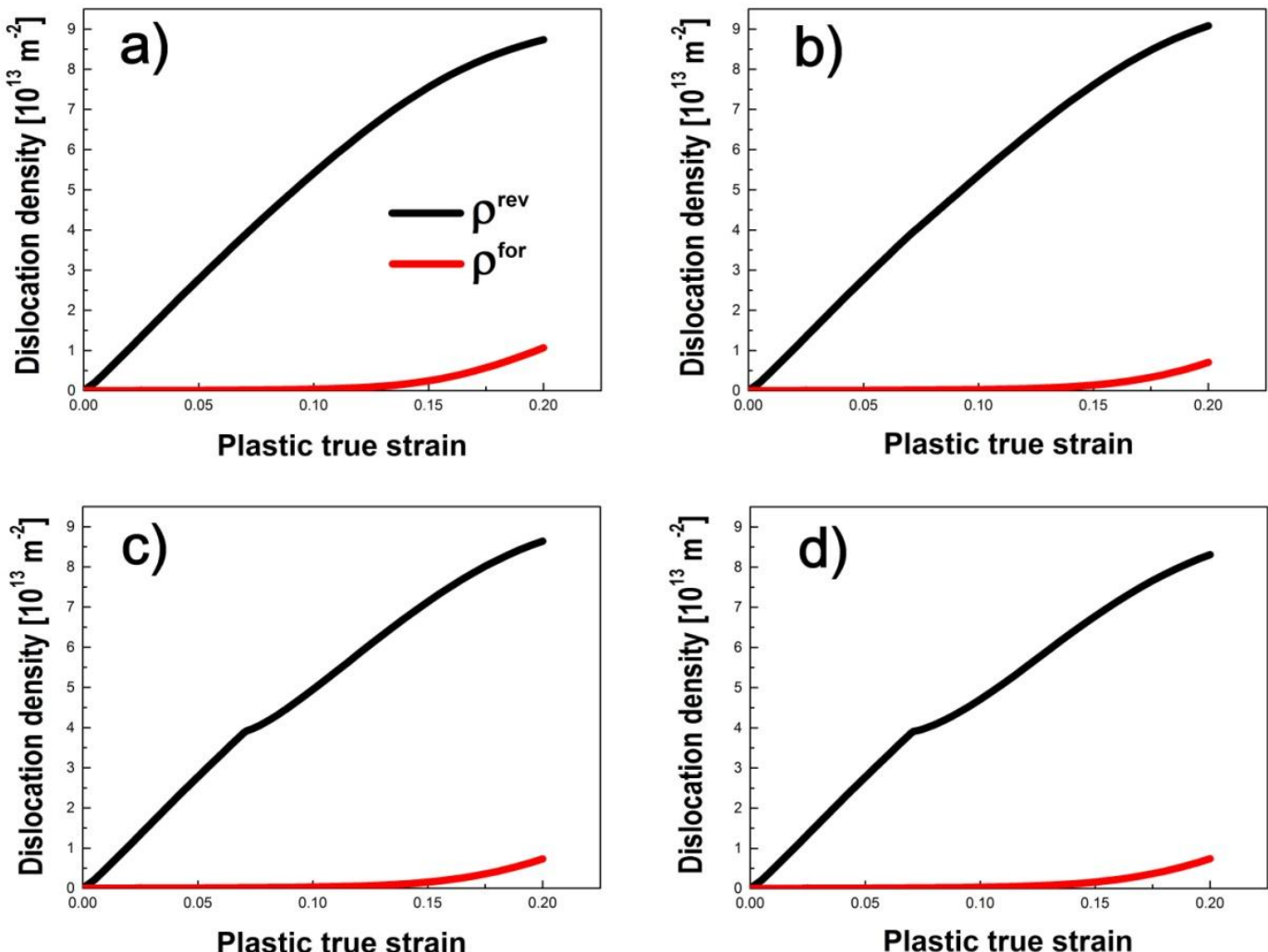

Fig. 6. Evolution of forward and reversible dislocation densities for the reloading along $0^{\circ}$ (a), $30^{\circ}$ (b), $60^{\circ}$ (c) and $90^{\circ}$ (d) from RD. 

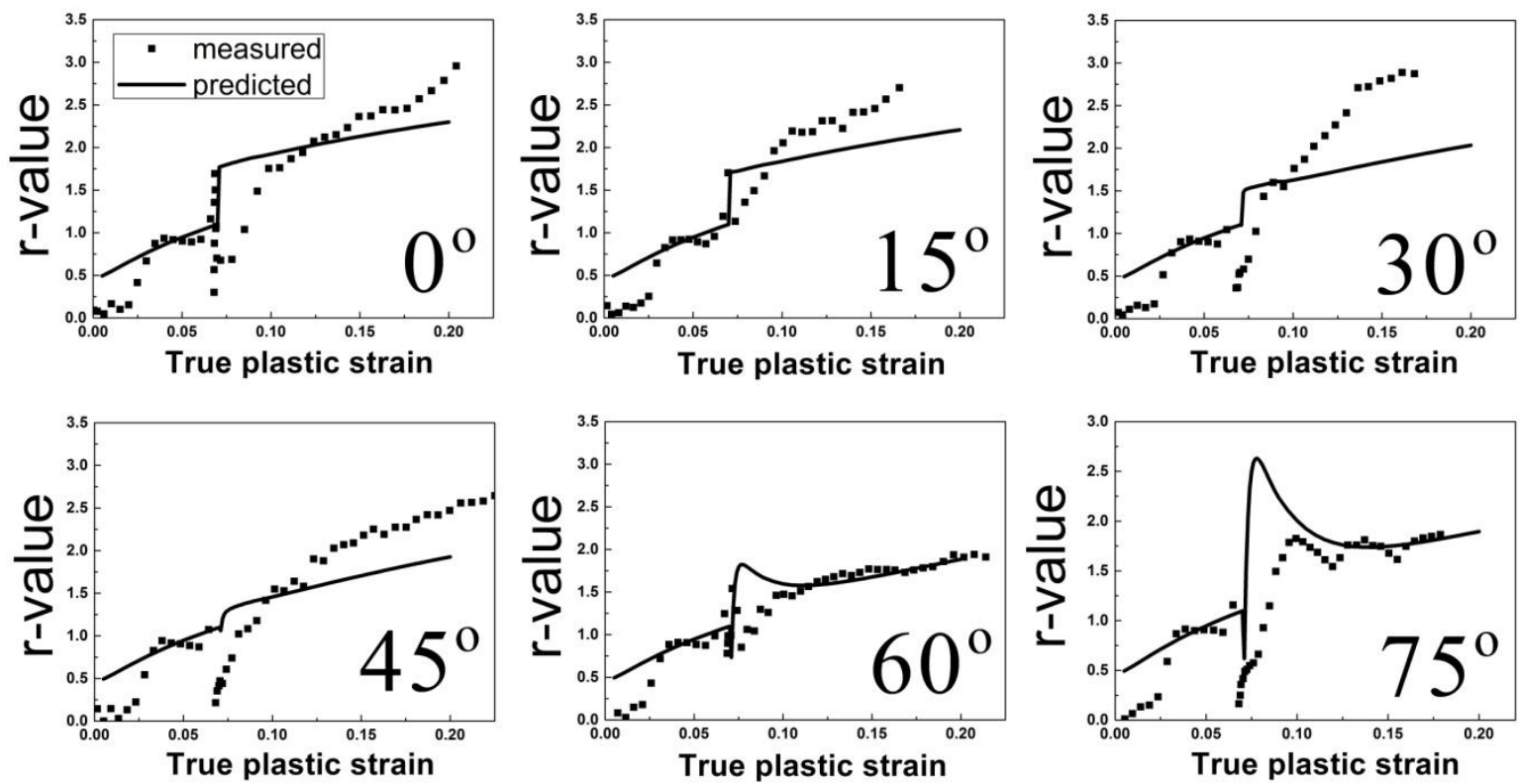

Fig. 7. Measured and predicted evolution of Lankford coefficients for different directions. 
The newly introduced back-stress law (Eq. 11) allows the prediction of the Bauschinger effect by reducing the CRSSs of the slip systems that experience reversals after reloading. The contribution of this mechanism is highlighted in Fig. 8, where the predicted stress-strain curves with and without back-stress are presented. The effect becomes more marked as the reload angle deviates more from the pre-load condition and reversibility of dislocations is more likely to occur on previously active systems. Fig. 9 presents the effect of back-stress on the relative activities (Fig. 9a), r-value (Fig. 9b) and dislocation density evolution (Fig. 9c) for reloading at $90^{\circ}$ from $\mathrm{RD}$, the case where this effect is more pronounced. It can be seen that accounting for the back-stress effect leads to a decrease in basal slip activity and an increase in prism slip activity during a reload transition of about 3\% strain (Fig. 9a). As mentioned in section 3.3, the back-stress effect depends on the accumulated reversible dislocation density before the shear on the slip system is reversed. The basal systems accumulate higher reversible dislocation density than the prismatic systems because they are relatively more active before reload. Therefore, if the shear on a basal slip system is reversed, the back-stress law (Eqs. 11 and 12) will lead to a relatively larger reduction of the CRSS of this system and a potentially higher relative activity for basal slip. However, the relative activity also depends on the grain orientation. In the present work, for the first reloading step along $90^{\circ}$ from RD, the CRSSs in the reversed basal slips decreases (in average) about $40 \%$ because of the back-stress effect, whereas the ones in the prismatic slips decrease about $28 \%$. At the same time, for the grain orientations at reloading, the reversals of prismatic slip occur about $2 \sim 3$ times more frequently than the ones of basal slip over the whole aggregate. This is responsible for the decrease in the relative basal activity (Fig. 9a), and the increase of r-value (Fig. 9b) at reloading. The back-stress effect mostly affects the flow stress transition. The associated dislocation annihilation does not affect the total dislocation density appreciably (Fig 9c), and the associated changes in system activity do not affect the predicted texture either. 


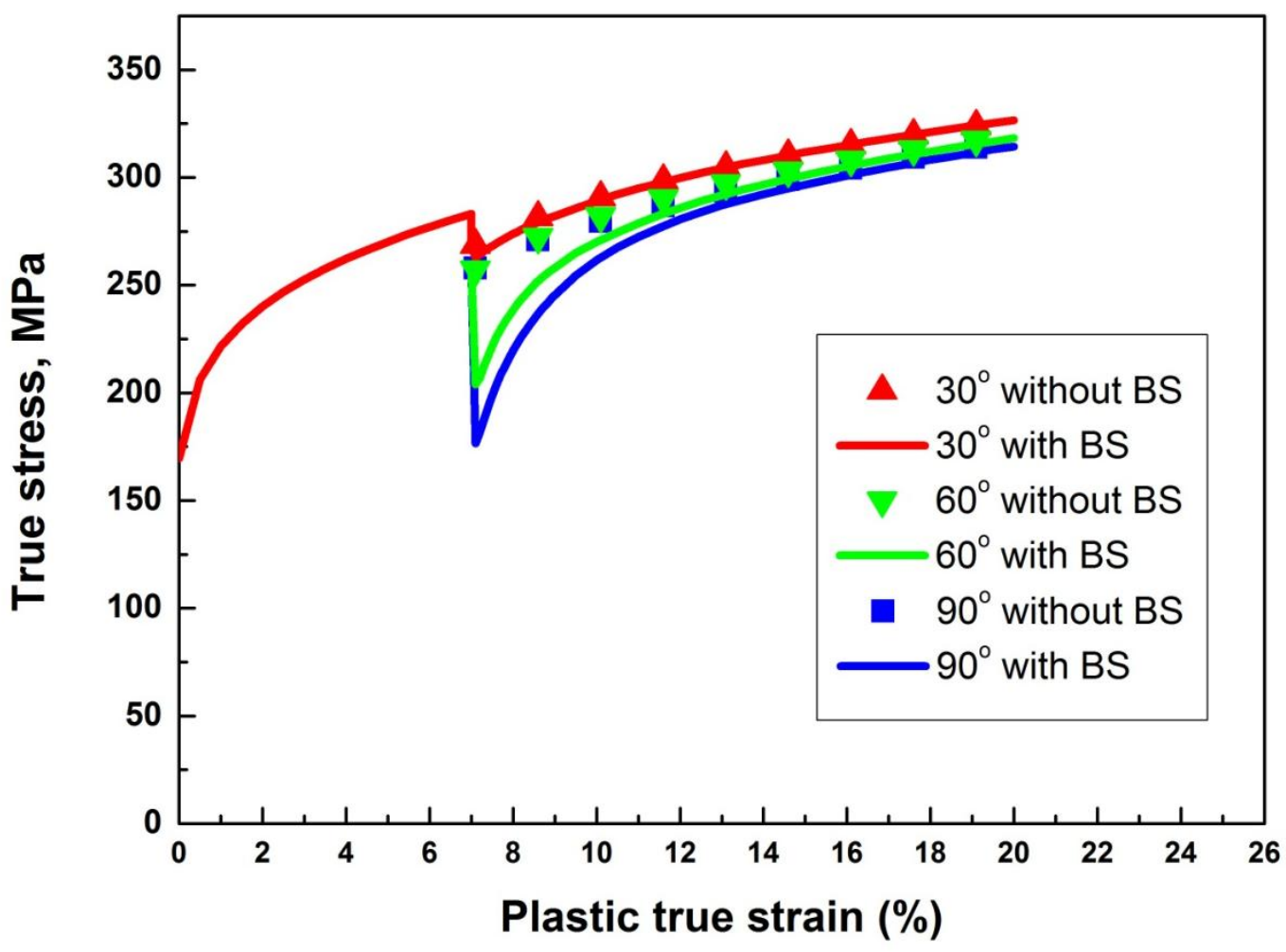

Fig. 8. Contribution of back-stress to the strain-stress response for different reloading directions. 

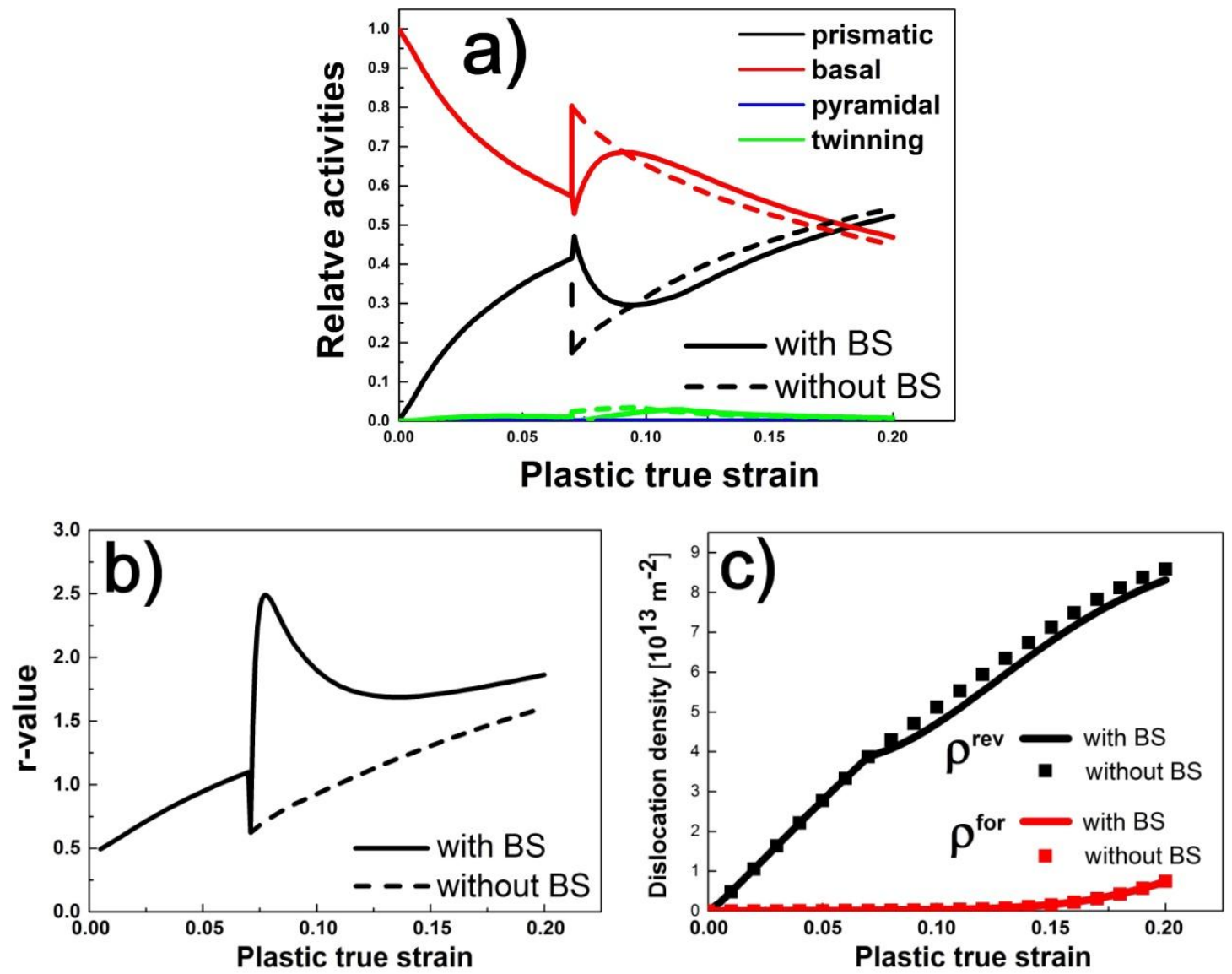

Fig. 9. Contribution of back-stress to the evolutions of relative activities (a), r-value (b) and dislocation density (c) for the reloading along $90^{\circ}$ from RD. 


\section{Conclusion}

Two-step tension tests were carried out on rolled $\mathrm{Mg}$ sheet with different reloading directions. The experimental yield stress in the second step decreases as the reload angle with respect to the RD increases. Such an effect was shown to be mostly due to the back-stress associated with the dislocations created during pre-loading, with a minor contribution of the anisotropy associated with texture. In this work, a microstructure-based hardening model (RGBV model) previously applied to low carbon steel by Kitayama et al (2013), is adapted, improved and applied to $\mathrm{Mg}$ alloy. This model is able to track the evolution of reversible and forward dislocation densities (accumulation and annihilation) for each slip system throughout the deformation process. A new formulation of back-stress is added into this approach to predict the Bauschinger effect occurring during the initial several percents of reloading strain. The complex slip-slip interaction associated with the various active modes in HCP structures is also considered. It is shown that this model, embedded in the VPSC framework, well captures the experimental results in terms of flow stress, reload yield stress, texture and, to a reasonable extent, Lankford coefficient evolutions.

\section{ACKNOWLEDGMENTS}

CT acknowledges support from US Department of Energy, Office of Basic Energy Science, Division of Materials Science and Engineering, Project FWP 06SCPE401DOE-BES. WW, MB, JG and FB, acknowledge the financial support of Portuguese Foundation of Science and Technology projects PTDC/EME-TME/105688/2008, PEST-C/EME/UI0481/2011 and PESTC/EME/UI0481/2013. 


\section{Reference:}

Aghababaei, R., Joshi, S.P., 2014. Micromechanics of tensile twinning in magnesium gleaned from molecular dynamics simulations. Acta Mater. 69, 326-342.

Agnew, S., Duygulu, O., 2005. Plastic anisotropy and the role of non-basal slip in magnesium alloy AZ31B, Int. J. Plasticity 21 (6), 1161-1193.

Agnew, S.R., Yoo, M.H., Tomé, C.N., 2001. Application of texture simulation to understanding mechanical behavior of $\mathrm{Mg}$ and solid solution alloys containing Li or Y, Acta Mater. 49, 42774289.

Barlat, F., Gracio, J.J., Lee, M.G., Rauch, E.F., Vincze, G., 2011. An alternative to kinematic hardening in classical plasticity. Int. J. Plasticity 27, 1309-1327.

Bauschinger, J., 1881. Ueber die VeraËnderung der Elasticita Ëtsgrenze und des ElasticitaËtsmoduls verschiedener Metalle. Civiling N.F. 27, 289-348.

Bertin, N., Tomé, C.N., Beyerlein, I.J., Barnett, M.R., Capolungo, L., 2014. On the strength of dislocation interactions and their effect on latent hardening in pure Magnesium. Int. J. Plasticity, accepted July 2014.

Beyerlein, I. J., Tomé, C.N., 2007. Modeling transients in the mechanical response of copper due to strain path changes, Int. J. Plasticity, 23, 640-664.

Brown, D.W., Agnew, S.R., Bourke, M.A.M., Holden, T.M., Vogel, S.C., Tomé, C.N., 2005. Internal strain and texture evolution during deformation twinning in magnesium, Mater. Sci. Eng. A 399, 1-12.

Brown, D.W., Beyerlein, I.J., Sisneros, T.A., Clausen, B., Tomé, C.N., 2012. Role of twinning and slip during compressive deformation of beryllium as a function of strain rate. Int. J. Plasticity 29, 120-135.

Cáceres, C.H., Lukáč, P., 2008. Strain hardening behaviour and the Taylor factor of pure magnesium. Philos. Mag. 88, 977-989.

Cáceres, C.H., Blake, A.H., 2007. On the strain hardening behaviour of magnesium at room temperature. Mater. Sci. Eng. A 462, 193-196.

Capolungo, L., 2011. Dislocation junction formation and strength in magnesium. Acta Mater. 59, 2909-2917.

Chapuis, A., Wang, B., Liu, Q., 2014. A comparative study between uniaxial compression and plane strain compression of $\mathrm{Mg}-3 \mathrm{Al}-1 \mathrm{Zn}$ alloy using experiments and simulations. Mater. Sci. Eng. A 597, 349-358.

Christodoulou N., 1989. Evolution of the Bauschinger effect in tension and compression in Zircaloy-2. Acta Metall. 37, 529-539.

Essmann, U., Mughrabi, H., 1979. Annihilation of dislocations during tensile and cyclic deformation and limits of dislocation densities. Phil. Mag. A 40, 731-756.

Feaugas, X., 1999. On the origin of the tensile flow stress in the stainless steel AISI 316L at 300 $\mathrm{K}$ : back stress and effective stress. Acta Mater. 47, 3617-3632. 
Hielscher, R., Schaeben, H., 2008. A novel pole figure inversion method: specification of the MTEX algorithm. J. Appl. Crystallography, 41, 1024-1037.

Jain, A., Agnew, S.R., 2007. Modeling the temperature dependent effect of twinning on the behavior of magnesium alloy AZ31B sheet. Mater. Sci. Eng. A 462 (2007) 29-36.

Kitayama, K., Tomé C.N., Rauch E.F., Gracio, J.J., Barlat, F., 2013. A crystallographic dislocation model for describing hardening of polycrystals during strain path changes. Application to low carbon steels, Int. J. Plasticity, 46, 54-69.

Khan, A. S., Pandey, A., Gnäupel-Herold ,T., Mishra , R.K., 2011. Mechanical response and texture evolution of AZ31 alloy at large strains for different strain rates and temperatures, Int. J. Plasticity, 27(5), 688-706.

Khosravani, A., Scott, J., Miles, M.P., Fullwood, D., Adams, B.L., Mishra, R.K., 2013. Twinning in magnesium alloy AZ31B under different strain paths at moderately elevated temperatures, Int. J. Plasticity, 45, 160-173.

Knezevic, M., McCabe, R.J., Lebensohn, R.A., Tomé, C.N., Liu, C., Lovato, M.L., Mihaila, B., 2013. Integration of self-consistent polycrystal plasticity with dislocation density based hardening laws within an implicit finite element framework: Application to low-symmetry metals, Journal of the Mechanics and Physics of Solids, 61, 2034-2046

Kocks, U.F., Mecking, H., 2003. Physics and phenomenology of strain hardening: the FCC case. Prog. Mater. Sci. 48, 171-273.

Kubin, L., Devincre, B., Hoc, T., 2008. Modeling dislocation storage rates and mean free paths in face-centered cubic crystals. Acta Mater. 56, 6040-6049.

Lebensohn, R.A., Tomé, C.N., 1993. A self-consistent anisotropic approach for the simulation of plastic deformation and texture development of polycrystals-Application to zirconium alloys. Acta Metall. Mater. 41, 2611-2624.

Lebensohn, R.A., Tomé, C.N., Ponte Castañeda, P., 2007. Self-consistent modeling of the mechanical behavior of viscoplastic polycrystals incorporating intragranular field fluctuations. Philos. Mag. 87, 4287-4322.

Lee, M.G., Lim, H., Adams, B.L., Hirth, J.P., Wagoner, R.H., 2010. A dislocation density-based single crystal constitutive equation. Int. J. Plasticity 26, 925-938.

Li D.S., Ahzi, S., M'Guil, S., Wen, W., Lavender, C., Khaleel, M.A., 2014. Modeling of deformation behaviour and texture evolution in magnesium alloy using the intermediate $\phi$ model, Int. J. Plasticity 52, 77-94.

Ma, Q., Kadiri, H. El, Oppedal, A.L., Baird, J.C., Li, B., Horstemeyer, M.F., Vogel, S.C., 2012. Twinning effects in a rod-textured AM30 Magnesium alloy, Int. J. Plasticity, 29, 60-76.

Mayama, T., Noda, M., Chiba, R., Kuroda, M., 2011. Crystal plasticity analysis of texture development in magnesium alloy during extrusion, Int. J. Plasticity, 27, 1916-1935.

Molinari, A., Canova, G.R., Ahzi, S., 1987. A self-consistent approach of the large deformation polycrystal viscoplasticity. Acta metall. 35, 2983-2994.

Mughrabi, H., Ungar, T., Kienle, W., Wilkens, M., 1986. Long-range internal stresses and asymmetric X-ray line-broadening in tensile-deformed [001]-orientated copper single crystals. Phil. Mag. A 53, 793-813. 
Neil, C. J., Agnew, S. R., 2009. Crystal plasticity-based forming limit prediction for non-cubic metals: Application to Mg alloy AZ31B, Int. J. Plasticity, 25, 379-398.

Oppedal, A.L., Kadiri, H. El., Tomé, C.N., Kaschner, G.C., Vogel, S. C., Baird, J.C., Horstemeyer, M.F., 2012. Effect of dislocation transmutation on modeling hardening mechanisms by twinning in magnesium, Int. J. Plasticity, 30-31, 41-61.

Paliwal, M., Jung, I.H., 2014. Microstructural evolution in Mg-Zn alloys during solidification: An experimental and simulation study. J. Cry. Growth 394, 28-38

Parks, D.M., Ahzi, S., 1990. Polycrystalline plastic deformation and texture evolution for crystals lacking five independent slip systems. J. Mech. Phys. Solids 38, 701-724.

Peeters, B., Kalidindi, S.R., Van Houtte, P., Aernoudt, E., 2000. A crystal plasticity based work hardening/softening model for BCC metals under changing strain paths. Acta Mater. 48, 2123 2133.

Peeters, B., Bacroix, B., Teodosiu, C., Van Houtte, P., Aernoudt, E., 2001. Workhardening/softening behaviour of BCC polycrystals during changing strain paths: I. An integrated model based on substructure and texture evolution, and its prediction of the stressstrain behaviour of an IF steel during two-stage strain paths. Acta Mater. 49, 1607-1619.

Piao, K., Lee, J.K., Kim, J.H., Kim, H.Y., Chung, K., Barlat, F., Wagoner, R.H., 2012. A sheet tension/compression test for elevated temperature, Int. J. Plasticity, 38, 27-46.

Proust, G., Tomé, C.N., Kaschner, G.C., 2007. Modeling texture, twinning and hardening evolution during deformation of hexagonal materials. Acta Mater. 55, 2137-2148.

Proust, G., Tomé, C.N., Jain, A., Agnew, S.R., 2009. Modeling the effect of twinning and detwinning during strain-path changes of magnesium alloy AZ31. Int. J. Plast. 25, 861-880.

Rauch, E.F., Gracio, J.J., Barlat, F., 2007. Work-hardening model for polycrystalline metals under strain reversal at large strain. Acta Mater. 55, 2939-2948.

Rauch, E.F., Gracio, J.J., Barlat, F., Vincze, G., 2011. Modelling the plastic behavior of metals under complex loading conditions. Modell. Sim. Mater. Sci. Eng. 19 (035009), 1-18.

Rossiter, J., Brahme, A., Simha, M.H., Inal, K., Mishra, R., 2010. A new crystal plasticity scheme for explicit time integration codes to simulate deformation in 3D microstructures: effects of strain path, strain rate and thermal softening on localized deformation in the aluminum alloy 5754 during simple shear. Int. J. Plasticity 26, 1702-1725.

Sánchez-Martín, R., Pérez-Prado, M.T., Segurado, J., Bohlen, J., Gutiérrez-Urrutia, I., J. Llorca Molina-Aldareguia, J.M., 2014. Measuring the critical resolved shear stresses in Mg alloys by instrumented nanoindentation. Acta Mater. 71, 283-292.

Schoenfeld, S.E., Ahzi, S., Asaro, R.J., 1995. Elastic-plastic crystal mechanics for low symmetry crystals. J. Mech. Phys. Solids 43, 415-446.

Schwartz, J., Fandeur, O., Rey, C., 2013. Numerical approach of cyclic behaviour of 316LN stainless steel based on a polycrystal modelling including strain gradients. Int. J. Fatigue 55, 202212.

Stout, M.G., Rollett, A.D., 1990. Large strain Bauschinger effects in FCC metals and alloys. Metall. Trans. 21A, 3201-13. 
Tomé, C.N., Lebensohn, R.A., Kocks, U.F., 1991. A model for texture development dominated by deformation twinning: application to zirconium alloys. Acta Metal. Mater. 39, 2667-2680.

Wang, Y.N., Huang, J.C., 2003. Texture analysis in hexagonal materials. Mater. Chem. Phys. 81, 11-26.

Wang, H., Raeisinia, B., Wu, P.D., Agnew, S.R., Tomé, C.N., 2010. Evaluation of selfconsistent polycrystal plasticity models for magnesium alloy AZ31B sheet. Int. J. Solids Struct. 47, 2905-2917.

Wang, H., Wu, P.D., Boyle, K.P., Neale, K.W., 2011. On crystal plasticity formability analysis for magnesium alloy sheets. Int. J. Solids Struct. 48, 1000-1010.

Wang, H., Wu, P.D., Wang, J., 2013. Modeling inelastic behavior of magnesium alloys during cyclic loading-unloading. Int. J. Plasticity 47, 49-64.

Xue, Q., Beyerlein, I.J., Alexander, D.J., Gray III, G.T., 2007. Mechanisms for initial grain refinement in OFHC copper during equal channel angular pressing. Acta Mater. 55, 655-668.

Yoon, J.W., Barlat, F., Gracio, J.J., Rauch, E., 2005. Anisotropic strain hardening behavior in simple shear for cube textured aluminum alloy sheets. Int. J. Plasticity 21, 2426-2447.

Zhang, J., Yu, Q., Jiang, Y., Li, Q., 2011. An experimental study of cyclic deformation of extruded AZ61A magnesium alloy, Int. J. Plasticity, 27, 768-787. 


\section{FIGURE CAPTIONS}

Fig. 1. Experimental true stress-true strain curves for tension-tension tests with different reloading angles from $\mathrm{RD}$

Fig. 2. Initial texture of rolled $\mathrm{Mg}$ sheet.

Fig. 3. Comparison of predicted and measured true strain-true stress curves for different reloading directions.

Fig. 4. Predicted and experimental texture after $7 \%$ pre-strain (a) and after $13 \%$ reloading for $0^{\circ}$ (b), $45^{\circ}$ (c) and $90^{\circ}$ (d) from RD. PLD=Pre-loading direction. RLD=reloading direction. Intensity lines are $0.5,1,2,4,8 \mathrm{mrd}$.

Fig. 5. Relative slip/twinning activities for the reloading along $0^{\circ}$ (a), $30^{\circ}$ (b), $60^{\circ}$ (c) and $90^{\circ}$ (d) from RD.

Fig. 6. Evolution of forward and reversible dislocation densities for the reloading along $0^{\circ}$ (a), $30^{\circ}(\mathrm{b}), 60^{\circ}(\mathrm{c})$ and $90^{\circ}$ (d) from RD.

Fig. 7. Measured and predicted evolution of Lankford coefficients for different directions.

Fig. 8. Contribution of back-stress to the strain-stress response for different reloading directions.

Fig. 9. Contribution of back-stress to the evolutions of relative activities (a), r-value (b) and dislocation density (c) for the reloading along $90^{\circ}$ from RD.

Table 1. Values of the latent hardening matrix $\alpha^{s s^{\prime}}$ for various slip/slip interactions

Table 2. Parameters of the RGBV model and PTR scheme for Mg alloy. 